\title{
West Indian punctate keratopathy in a Caucasian
}

\author{
C. A. BUCKLEY AND A. J. BRON \\ From the Nuffield Laboratory of Ophthalmology and Oxford Eye Hospital, Oxford
}

SUMMARY We present a patient with the picture of corneal white spots, very similar in morphology to those described in native West Indians, occurring here in a Caucasian. Their aetiology remains obscure.

The case is reported of a female Caucasian with white corneal spots described previously only in native West Indians. We believe this to be the only report of this finding in a Caucasian.

\section{Case report}

A 41-year-old white female presented to us in October 1978 with the complaint of intermittent redness and grittiness of both eyes for the previous 3 months. This had begun just before she had left Rhodesia at a time when she was nursing sick children, some with conjunctivitis. Her symptoms had responded favourably to betamethasone drops and she was currently asymptomatic.

She was born in Trinidad, West Indies, of white parents. Her schooling had been in Barbados from the age of 12 to 17 , and from 18 to 24 years of age she had lived in England. From there she emigrated to Africa, where she lived until the age of 40 . She had lived in Nyasaland for 18 months and had then moved to Rhodesia, initially in the Zambesi River Valley, later in the Low Veld, and then in Malsetter (East Rhodesia). She had lived among the black tribes as her husband was involved in tsetse fly control by aerial spraying. In August 1978 she returned to England.

She had filariasis diagnosed by blood examination a few years ago, and was treated with 2 courses of penicillin for primary syphilis on her return to England.

Examination of the patient showed the lesions to be confined to the left cornea. Both eyes were white, and there was no conjunctival reaction. The visual acuity, anterior chambers, irides, intraocular pressures, and fundi were all normal. The right cornea was normal.

The left cornea showed three small dense, white,

Correspondence to Dr C. A. Buckley, The Eye Hospital, Walton Street, Oxford OX2 6AN. round, subepithelial spots clustered together in the inferonasal quadrant. The epithelium was intact and showed no staining areas with fluorescein or Bengal rose dyes. The deeper stromal layers and endothelium beneath the spots were normal. Corneal sensation was not impaired, and corneal thickness was in the normal range.

Figs. 1-3 show the spots. Each was approximately $0.2 \mathrm{~mm}$ in diameter, and high-power slit-lamp examination showed each dense area to have a coarse granular appearance, surrounded by a halo of stromal haze about as wide as the opacity itself.

\section{Discussion}

The dense white corneal spots described here in a Caucasian female appear to resemble those white spots in native West Indians reported by Rice et al. ${ }^{1}$ As in their cases, the condition was a chance finding in a patient with symptoms seemingly unrelated to the presence of the spots. A diagnosis of West Indian spot was made before her place of birth had been established.

Unfortunately we have no electron microscopic confirmation of the identity of these spots.

The lesions in our case were confined to the interpalpebral cornea. In the paper of Rice et al. ${ }^{1}$ trauma was mooted as a possible aetiological factor. Until now these lesions have been described only in West Indians, and in our patient also it is likely that the lesions were acquired during her domicile in the West Indies between birth and the age of 18 years. During that time it appears that she lived a life, in terms of exposure to trauma, no different from that of a British subject schooled in England.

Early ocular onchocerciasis may be seen as discoid or nummular opacities which are clear-cut, permanent, anterior stromal scars, usually in the interpalpebral zone and close to the limbus, and may be preceded or accompanied by a nonspecific 


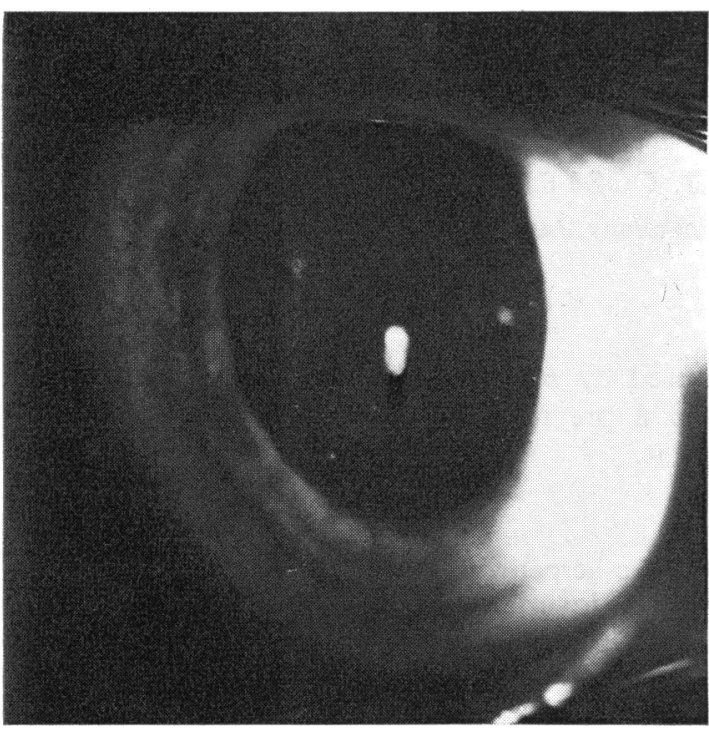

Fig. 1 West Indian spots, left cornea. Scleral scatter photograph of spots.

allergic type of conjunctivitis. ${ }^{2}$ As our patient had not, so far as we know, lived in an endemic onchocerciasis zone, and as her filariasis was apparently diagnosed by a blood smear, it seems that she is unlikely to have teen suffering from onchocerca infestation, and thus her corneal opacities cannot easily be attributed to this disease, nor did they have the morphology of onchocercal 'snow-flake' lesions.

There is also no corneal evidence of a syphilitic infection, and the appearances were not those of adenovirus or chlamydial keratitis.

Although the patient had been exposed to and had symptoms of conjunctivitis, the lesions were not active at the time of examination and did not vary in appearance over a period of 12 months.

The authors thank Mr D. Barbour for photographic preparation and Miss J. E. Sawyer for typing the manuscript.

\section{References}

1 Rice NSC, Jones BR, Ashton N. Punctate keratopathy of West Indians. Br J Ophthalmol 1968; 52: 865-75.

2 Duke-Elder S. System of Ophthalmology. London: Kimpton, 1965: 8: 411

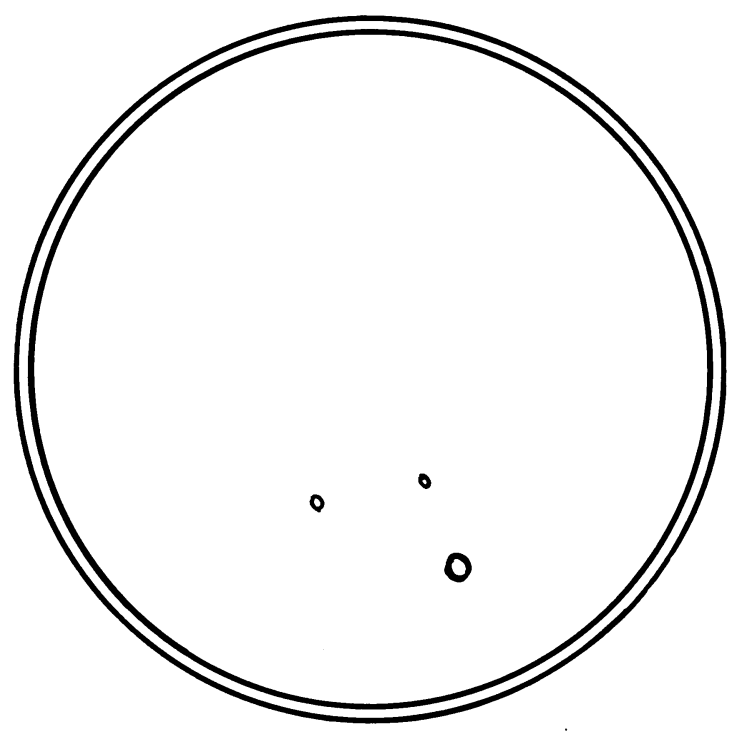

Fig. 2 Distribution of spots on cornea.

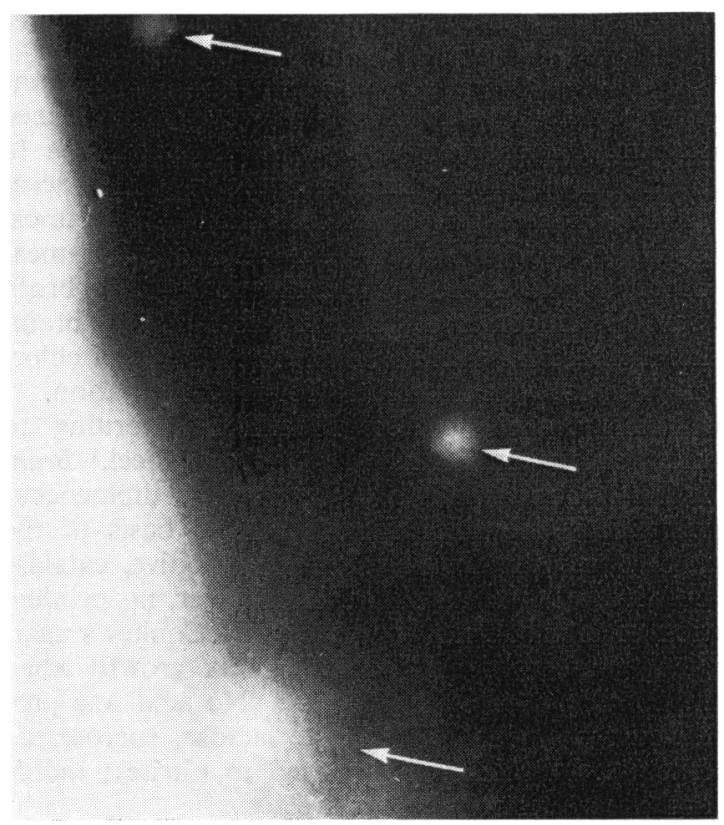

Fig. 3 Macrophotograph of West Indian spots $(\times 30)$. 